\title{
Recommendations for HER2 testing in the UK
}

\author{
I O Ellis, M Dowsett, J Bartlett, R Walker, T Cooke, W Gullick, B Gusterson, E Mallon, \\ P Barrett Lee
}

Department of
Histopathology, City
Hospital, Hucknall
Road, Nottingham
NG5 1PB, UK
I O Ellis
Department of
Biochemistry, The
Royal Marsden
Hospital, Fulham
Road, London SW3 6JJ,
UK
M Dowsett
University
Department of
Surgery, Glasgow
Royal Infirmary, Level
II, Queen Elizabeth
Building, Alexandra
Parade, Glasgow
G31 2ER, UK
J Bartlett
T Cooke

University of Leicester, Department of Pathology, School of Medicine, Robert Kilpatrick Clinical Sciences Building, Leicester Royal Infirmary, PO Box 65, Leicester LE2 7LX, UK $\mathrm{R}$ Walker

Department of Biosciences, The University of Kent at Canterbury, Canterbury, Kent CT2 7NJ, UK W Gullick

Department of Pathology, Western Infirmary, Dumbarton Road, Glasgow G11 6NT, UK

B Gusterson E Mallon

Verlindre Hospital, Whitchurch, Cardiff DF4 7XL, UK

P Barrett Lee ian.ellis@nottingham.ac.uk

Correspondence to: Dr Ellis

Accepted for publication 12 October 2000

\begin{abstract}
Determining the HER2 status of breast carcinomas is a prerequisite for the use of the monoclonal antibody trastuzumab (Herceptin $\left.{ }^{\circledR}\right)$, which has recently been licensed for the treatment of metastatic disease. This necessitates a test based on archival material. The preferred analyses are immunohistochemistry with fluorescent in situ hybridisation (FISH) as a follow up test for ambiguous results. Guidelines have been developed for standardised, well controlled procedures for the provision of reliable results. A group of three reference laboratories has been established to provide advice, quality assurance, and materials, where needed. (F Clin Pathol 2000;53:890-892)
\end{abstract}

Keywords: HER2; breast cancer; Herceptin ${ }^{\circledR}$

The humanised anti-HER-2/neu (also known as c-erbB-2, further denoted HER2) monoclonal antibody trastuzumab (Herceptin ${ }^{\mathbb{B}}$ ) can be used to treat women with HER2 positive metastatic breast cancer. ${ }^{1}$ Establishing tumour HER2 status is a prerequisite for the use of trastuzumab. ${ }^{2}$ However, a widely accepted and standardised test for HER2 status is not currently available. ${ }^{3}$ These recommendations have been designed to give notice of the availability of a reference laboratory service and to give advice on methodology for local testing to ensure that HER2 testing results are accurate and reliable, regardless of the test that is used.

\section{General principles}

SUITABLE SAMPLES

Formalin fixed, paraffin wax embedded tumour tissue samples are appropriate for assay. ${ }^{4}$ Other methods of tissue fixation can adversely affect reactivity.

APPROPRIATE LABORATORY ASSAY METHODS Immunohistochemistry (IHC) and fluorescent in situ hybridisation (FISH) ${ }^{5}$ are the techniques recommended for determining HER2 status. We do not intend to be prescriptive about methodology or the antibodies suitable for ICH because there is no substantive evidence to support the exclusive use of one IHC testing method or reagent. For immunohistochemical HER2 testing, comprehensive standardisation of methodology and the inclu- sion of validated controls are mandatory. The Hercep Test ${ }^{\circledR}$ meets these criteria, but owing to cost considerations, other IHC tests and antibodies other than the polyclonal antiserum used in this test may be used (see recommendations regarding the use of controls and test validation below).

FISH testing for HER2 should meet the following criteria: (1) the inclusion of a chromosome 17 control to allow for correction of the HER2 signal number for chromosome 17 aneusomy (seen in over $50 \%$ of cases); (2) comprehensive standardisation of methodology; and (3) validated controls. Although there are several commercial systems available for FISH HER2 testing, the PathVysion ${ }^{\mathrm{TM}}$ assay (Vysis, Richmond, Surrey, UK) is recommended for diagnostic testing because it meets the above criteria. Owing to cost considerations, other probes and in house prepared probes may be use, but care must be taken to ensure that these are correctly validated (see recommendations below).

Currently, the other available HER2 testing techniques (polymerase chain reaction, enzyme linked immunosorbent assay, Southern blotting) should be used for research only. Standard protocols should be developed and applied for local IHC or FISH HER2 tests. These should be validated using standardised commercial kit assays or local assay methodologies that have been validated against the well characterised tissue sets that are available from the reference laboratories (see below).

\section{CONTROLS}

The inclusion of controls of known HER2 status in all HER2 tests is required to ensure test accuracy. One positive and one negative control of known immunoreactivity and gene signal number are recommended as a minimum; controls producing results close to important decision making points should also be considered.

The reference laboratories can validate locally produced controls by FISH and HercepTest if required.

Tissue array blocks containing multiple tissue samples of known HER2 status characterised by FISH and IHC are being developed by the reference laboratories to facilitate test validation. 


\section{Immunohistochemistry}

FOR ALL IHC TESTS

Antigen retrieval processes should be standardised and follow strict protocols. The antibody used and its titre should be predefined. No single antibody has been consistently demonstrated to be superior in terms of specificity and sensitivity. At present, antibodies CB11 (Novocastra, Newcastle Upon Tyne, UK), TAB 250 (Zymed, San Francisco, California, USA), and A0485 (Dako, Ely, Cambridgeshire, UK) are the most widely used. Test conditions (temperature, exposure time, etc) should be standardised.

There is evidence that HER2 protein reactivity may deteriorate in fixed paraffin wax sections over a period of months. It is recommended that control block sections are prepared fresh or that storage time is restricted to $<3$ months unless testing to assess degradation has been conducted.

Test conditions should be optimised so that distinct moderate or strong membrane staining identifies FISH positive samples.

Laboratories not wishing to standardise in house methodology should consider using a commercial kit assay system such as the HercepTest.

Excessive antigen retrieval can be monitored by an evaluation of normal breast epithelial cells as an internal control. Should membrane reactivity be identified in the normal cell population, excessive antigen retrieval may have occurred. It is recommended that the assay be rejected and retested. Alternatively, some groups have recommended that scoring of the tumour cell population be modified by subtracting the degree of normal cell reactivity.

SCORING IHC

Only membrane staining of the invasive tumour should be considered when scoring IHC tests.

If the HercepTest is used, it is recommended that laboratories adhere strictly to the kit assay protocol and scoring methodology. Local modifications of techniques can lead to false positive and negative assay results. The scoring method provided with the kit uses a semiquantitative system based on the intensity and percentage of positive cells, giving a score range of 0 to $3+$. Samples scoring $3+$ are regarded as unequivocally positive and $0 / 1+$ as negative. Borderline $1+/ 2+$ and $2+$ require confirmation using another analysis system, preferably FISH.

Until there is more evidence based on patient response to treatment, we recommend using the HercepTest scoring method for tumours assessed using other IHC methods. We anticipate that simplified IHC scoring methods will be developed. For example, cases should be reported as positive for HER2 overexpression if $>10 \%$ of cells show moderate or strong complete membrane staining; cases that show equivocal/borderline reactivity should be retested, preferably using FISH.

Interobserver variation in the assessment of staining can lead to misclassification of HER2 status. Each individual should standardise scoring against known positive, negative, and borderline cases.

QUALITY ASSURANCE

Laboratories offering an IHC service are advised to participate in the UK NEQAS Immunohistochemistry External Quality Assurance (EQA) scheme.

Fluorescence in situ hybridisation (FISH) The Vysis PathVysion kit is recommended if FISH is performed because it meets the above mentioned criteria for proper HER2 FISH testing, with results being expressed as the ratio of HER2 signal to chromosome 17 signal. Tumours showing a ratio of $>2$ should be considered positive. Cut off values for HER2 gene amplification when chromosome 17 probes are not used have not been established.

\section{FOR ALL FISH TESTS}

There is no evidence that storage of blocks or slides leads to deterioration of signal. However, storage of cut sections from controls or samples for over six to 12 months should be avoided.

It is advisable to locate areas of invasive tumour using a serial section stained with haematoxylin and eosin and to use this to locate tumour areas to be scored after testing. Care should be taken to avoid areas of ductal carcinoma in situ, which can show amplification even where adjacent invasive tumour cells are negative.

Tissue digestion should be standardised to maintain nuclear morphology and should follow strict protocols. Nuclear structure should be evaluated before hybridisation and digestion, where appropriate, to preserve nuclear integrity. Hybridisation and washing steps should be standardised. Guidance can be provided by the reference laboratories.

It is recommended that commercially available probes are used. For systems using in house nick translated probes, attention should be given to batch variability of nick translation enzymes, etc.

Laboratories not wishing to use in house methods should consider using a commercial system such as the Vysis PathVysion kit.

\section{SCORING FISH}

For all test systems, the scoring protocol included in the PathVysion kit is recommended. The number of chromosome 17 and HER2 signals is scored for 60 cells, where possible from three distinct tumour fields, and the mean HER2 to chromosome 17 copy ratio is calculated. Where tumour heterogeneity is seen (1-2\% of cases), more cells from the amplified regions should be scored. Samples with $>2.0$ copies of HER2 for each chromosome 17 are considered to be amplified.

Interobserver variation should be assessed until good reproducibility is attained. For nonamplified samples, interobserver variation should be between $10 \%$ and $15 \%$. Variation increases with highly amplified samples, but is not critical where the ratio exceeds 4 . 
QUALITY ASSURANCE

To ensure adequate quality assurance, UK laboratories wishing to set up independent FISH testing are recommended to join the reference laboratories' EQA scheme.

\section{Reference laboratory service}

For the UK, three HER2 testing reference laboratories have been established in London, Nottingham, and Glasgow.

- Professor Mitch Dowsett, Department of Biochemistry, The Royal Marsden Hospital, Fulham Road, London SW3 6JJ, UK.

- Dr Ian Ellis, Department of Histopathology, City Hospital, Hucknall Road, Nottingham NG5 1PB, UK.

- Dr John Bartlett, University Department of Surgery, Glasgow Royal Infirmary, Level II, Queen Elizabeth Building, Alexandra Parade, Glasgow G31 2ER, UK.

These laboratories use validated IHC (HercepTest) and FISH techniques (PathVysion) to establish HER2 status, and also run an interlaboratory quality assurance scheme.
The service provided by the reference laboratories is available to all laboratories for advice and testing of HER2 status.

Test results will be provided within two weeks of sending to the reference laboratories. Laboratories that require data sooner or have in house procedures should conduct their testing according to the above guidelines.

Similar initiatives are expected in other countries where trastuzumab treatment is used.

1 Gralow J. Herceptin: New treatment modality. The Digital Breast Clinic 2000 http://www.digitalclinic.com/ herceptin.htm

2 Press MF, Hung G, Godolphin W, et al. Sensitivity of HER2/neu antibodies in archival tissue samples: potential source of errors in immunohistochemical studies of oncogene expression. Cancer Res 1994;54:2771-7.

3 Rampaul RS, Pinder SE, Gullick WJ, et al. Her 2 testing in breast cancer-methods of detection, clinical significance and future prospects. Crit Rev Oncol Hematol [[In press.]]

4 Wang S, Saboorian MH, Frenkel E, et al. Laboratory assessWang S, Saboorian MH, Frenkel E, et al. Laboratory assess-
ment of the status of Her-2/neu protein and oncogene in breast cancer specimens: comparison of immunohistochemistry assay with fluorescence in situ hybridisation assays. F Clin Pathol 2000;53:374-81.

5 Walker RA. The significance of histological determination of HER-2 status in breast cancer. The Breast 2000;9:130-3.

\section{PATHOLOGY INTERACTIVE 2000}

Do you know ... . ?

Pathology Interactive Volume 2 now offers:

Up to three CPD credits per review article

1 credit for reading the article and completing the associated questions

1 credit for additional reading, following up references

1 credit for making notes, identifying learning outcome or identifying further learning needs

CPD credits for working on articles outside your specialty

Pathology Interactive Volume 2 Issue 1 includes articles in:

Microbiology

Histopathology

Molecular pathology

Further issues in $\mathbf{2 0 0 0}$ will cover further articles in these specialties, plus:

Chemical pathology

Haematology

Immunology

Including case study and picture quiz formats

Pathology Interactive 2000 Volume 2, 4 issues (March, June, September, December) ISSN 1466 5743. Accredited by the Royal College of Pathologists

Subscription rate: $£ 75+$ VAT personal ${ }^{\star}, £ 150+$ VAT institutional (multiuser rate on application to the publisher).

Send orders to: BMJ Publishing Group, Journals Marketing Dept, PO Box 299, London WC1H 9TD, UK; fax credit card orders to: +44 (0)20 7383 6402; call subscriptions hotline +44(0)207383 6270; email orders to: subscriptions@bmjgroup.com

*ACP members receive Pathology Interactive with their copy of fournal of Clinical Pathology, as a membership benefit. 